\title{
Neonatal mortality risk prediction
}

Infant and child mortality is a health indicator of society in general, and a significant number of these deaths occurs at Neonatal Intensive Care Units.

Efforts aimed at preventing infant and child mortality are effective only if based on reasonably reliable information regarding death-related factors. Information is required on risk factors leading to such outcomes in order to prioritize interventions and plan their development, assess the effectiveness of interventions aimed specifically at risk factors, and observe national and international trends over time. ${ }^{1,2}$

Millennium Development Goal (MDG) 4, established by the World Health Organization (WHO), is to reduce child mortality by two thirds between 1990 and 2015, which accounts for an infant and child mortality rate of 8.5 per 1000 live births. ${ }^{3}$

Neonatal mortality ( $<28$ days old) is the main component of infant and child mortality $(<1$ year old) and accounts for approximately $50 \%$ of perinatal mortality. Given the progressive and sustained reduction in postnatal mortality (28365 days old), at present most efforts are focused on the perinatal period in order to reach the target of MDG 4.

The complexity and high costs related to neonatal and pediatric intensive care have led to a growing interest in developing measures to estimate the patient's condition severity. Although such scores may help to improve health care quality and reference mortality rates, it is important to fully understand their purpose and factors that influenced the performance of such risk scores so that they can be adequately compared and used more effectively. ${ }^{4}$

Every year, approximately four million children die in their first four weeks of life, and $99 \%$ of these deaths take place in developing countries. This is twice the number of deaths associated to HIV / AIDS. ${ }^{5}$

In this issue of Argentine Archives of Pediatrics (see page 213), Márquez-González, et al. describe a new neonatal mortality risk prediction scale in Mexico and its subsequent validation. This is an essential contribution and points the pathway to new advances.

An additional advantage of these studies is that they allow to draw conclusions on the studied population and the quality of care received (benchmarking). Thus, it is worth noting that, among pregnant women seen between 2010 and 2012 at the mother and child hospital where the study was conducted in Mexico City, the rate of antenatal corticosteroid treatment was extremely low (15\%) in spite of the well-known evidence on their benefit for newborn infants. For the sake of comparison, a study conducted by the WHO, which included 303842 pregnant women between 22 and 34 weeks of gestation, showed that the rate of antenatal corticosteroid administration reached 54\% (range: $16-$ 91\%). ${ }^{6}$ A recent Latin American multicenter study indicated that antenatal corticosteroid administration reduced the likelihood of neonatal mortality by $40 \%$ (unpublished data).

The study raises significant methodological challenges; the first one is gestational age estimate. Clinical methods (Capurro, Dubowitz, Ballard) may be helpful for newborn infants with a birth weight of more than $1500 \mathrm{~g}$, either in settings where the rate of an "uncertain" date of the last menstrual period is high or for research purposes. When the date of the last menstrual period is certain or reliable, it becomes an acceptable estimator of gestational age and may be improved by using ultrasound measurements during the first trimester of gestation. Such resources were overlooked by this study.

In addition, it would have been necessary to indicate the curve used to diagnose whether a newborn infant was small for gestational age. A recommendation for this is the INTERGROWTH$21^{\text {st }}$ study on fetal growth, which has a similar design to the WHO Multicentre Growth Reference Study. ${ }^{7}$

A second aspect to take into consideration is the estimation of the sample size for validation. An alternative to using the expected neonatal mortality rate is estimating the sample size for expected sensitivity and/or specificity. This way, for a sensitivity of 0.90 (with a lower confidence interval $>0.75$ ) and a prevalence of neonatal mortality $=0.10(10 \%)$, the number of cases was estimated to $b e=70$, and the number of controls $=630 .{ }^{8}$

A prediction scale seeks to identify subjects at risk of having a specific condition. The ideal attributes for an indicator to be used in these tests would be: a single, acceptable and lowcost measurement; a strong prediction or effect, combining adequate sensitivity and specificity; homogeneous, with small variation among 
studies; effective and efficient opportunity, intervention and diversion, with reduction of false classifications. This means that prediction tests are required to comply with a series of attributes, such as being simple, harmless, cheap, untroublesome and fast; in addition, it is fundamental to have a second-line test to exclude false positive and false negative results.

The proposed study shows certain limitations, such as a fast access to a clinical laboratory and the fact that upper values of specificity, and positive and negative odds ratios were achieved at the expense of a low sensitivity $(40 \%)$ and with scores above 15, which accounted for the worst conditions at birth, making us presume that they correspond to a minimum part of the population served ( $4 \%$ of survivors and $38 \%$ of deceased). The likelihood of survival, besides the large confidence intervals, proved that simple measures such as the birth weight, the Apgar score at 5 minutes after birth, and platelet count showed their maximum values, except for the lactate measurement, which in a new model could be replaced by glycemia.

Some pediatric and neonatal predictive models appear to be very adequate to make a comparison among institutions, with relatively brief data acquisition times, limited potential bias in relation to treatment, and reliance on diagnostic variables that allow to adjust the case mix. Other models are more adequate for use in clinical trials because they rely on physiological variables collected during a long period, in order to better establish the interaction between the functioning of body organ systems and specific therapeutic interventions in critically-ill patients. ${ }^{4}$

The fact that perinatal variables did not prove to be useful in the predictive model is not consistent with values described in the study; this may be attributed to the small sample size and to not having included the administration of antenatal corticosteroids to pregnant women, as in other scales. It would have also been important to indicate whether the administration of surfactants was accessible for all hospitalized newborn infants given their impact on neonatal mortality.

Birth asphyxia is the second condition associated to neonatal mortality $(8.7 / 100$ 000) following prematurity complications, as per a recent report. ${ }^{9}$ Changing the nomenclature from "birth asphyxia" to "neonatal mortality due to intrapartum complications" is welcome; however, there are many deaths due to a similar cause that go unreported, i.e., intrapartum fetal deaths, which were estimated to be 1.2 million globally, compared to 0.7 million neonatal deaths attributed to intrapartum events. These fetal deaths are an extension of asphyxia during labour and should be recorded and reported as part of neonatal mortality. These figures are even more alarming when considering that for every early neonatal death, a fetal death occurs. Such proportion exceeds deaths caused by vaccine preventable diseases and malaria combined. ${ }^{10}$

In countries where neonatal mortality rates are relatively low, its causes are probably attributed to prematurity and malformations, as opposed to asphyxia, tetanus and infections in countries with a high mortality rate.

Carlos Grandi, M.D.

Perinatal Epidemiology and Biostatistics

http:/ /dx.doi.org/10.5546/aap.2015.eng.196

\section{REFERENCES}

1. Bryce J, Boschi-Pinto C, Shibuya K, Black R, WHO Child Health Epidemiology Reference Group. WHO estimates of the causes of death in children. Lancet 2005; 365(9465):1147-52.

2. Liu L, Johnson H, Cousens S, Perin J, et al. Global, regional, and national causes of child mortality: an updated systematic analysis for 2010 with time trends since 2000 . Lancet 2012;379(9832):2151-61.

3. United Nations. General Assembly. United Nations Millennium Declaration. A/RES/55/2 edn. New York, NY: United Nations,2000. [Accessed on: February 3, 2015] Available at: http://www.un.org/millennium/ declaration/ares552e.pdf

4. Sacco Casamassima M, Salazar J, Papandria D, Fackler J, et al. Use of risk stratification indices to predict mortality in critically ill children. Eur J Pediatr 2014;173(1):1-13.

5. Katz J, Lee A, Kozuki N, Lawn J, et al. Mortality risk in preterm and small-for-gestational-age infants in lowincome and middle-income countries: a pooled country analysis. Lancet 2013;382(9890):417-25.

6. Vogel J, Souza J, Gülmezoglu A, Mori R, et al. Use of antenatal corticosteroids and tocolytic drugs in preterm births in 29 countries: an analysis of the WHO Multicountry Survey on Maternal and Newborn Health. Lancet 2014;384(9957):1869-77.

7. Villar J, Cheikh Ismail L, Victora C, Ohuma E, et al. International standards for newborn weight, length, and head circumference by gestational age and sex: the Newborn Cross-Sectional Study of the INTERGROWTH21st Project. Lancet 2014;384(9946):857-68.

8. Flahault A, Cadilhac M, Thomas G. Sample size calculation should be performed for design accuracy in diagnostic test studies. J Clin Epidemiol 2005;58(8):859-62.

9. GBD 2013 Mortality and Causes of Death collaborators. Global, regional, and national age-sex specific all-cause and cause-specific mortality for 240 causes of death, 1990 2013: a systematic analysis for the Global Burden of Disease Study 2013. Lancet 2015;385:117-71.

10. Cousens S, Blencowe H, Stanton C, Chou D, et al. National, regional, and worldwide estimates of stillbirth rates in 2009 with trends since 1995: a systematic analysis. Lancet 2011;377(9774):1319-30. 